\title{
Corrigendum
}

\section{Corrigendum to "Trajectory Tracking Control of Parallel Manipulator Based on Udwadia-Kalaba Approach"}

\author{
Chenming Li $\mathbb{D}$, ${ }^{1}$ Han Zhao, ${ }^{1}$ Shengchao Zhen $\left(\mathbb{D},{ }^{1,2}\right.$ Kang Huang, ${ }^{1}$ Hao Sun $\left(\mathbb{D},{ }^{1,2}\right.$ \\ Ke Shao, ${ }^{1}$ and Bin Deng ${ }^{1}$ \\ ${ }^{1}$ School of Mechanical Engineering, Hefei University of Technology, Hefei 230009, China \\ ${ }^{2}$ School of Mechanical Engineering, Georgia Institute of Technology, Atlanta 30332, USA \\ Correspondence should be addressed to Shengchao Zhen; zhenshengchao@qq.com
}

Received 23 May 2019; Accepted 10 June 2019; Published 12 September 2019

Copyright (C) 2019 Chenming Li et al. This is an open access article distributed under the Creative Commons Attribution License, which permits unrestricted use, distribution, and reproduction in any medium, provided the original work is properly cited.

In the article titled "Trajectory Tracking Control of Parallel Manipulator Based on Udwadia-Kalaba Approach" [1], there were errors in the author list and missing acknowledgments that have been described below and corrected in place. Dr. Shengchao Zhen, who suggested the idea of this article, was missing from the authors' list. Moreover, the second affiliation was missing.

Additionally, Dr. Chenming Li was incorrectly listed as the corresponding author. The corresponding author is Dr. Shengchao Zhen.

Finally, an acknowledgment has been added as follows:

The theoretical foundation of this paper is consistent with (1) National Natural Science Foundation of China (51505116), (2) the Fundamental Research Funds for the Central Universities (JZ2016HGTB0716), (3) Natural and Science Foundation of Anhui Province (1508085SME221), and (4) China Postdoctoral Science Foundation (2016M590563). In addition, the project is supported by these foundations.

\section{References}

[1] H. Zhao, C. Li, K. Huang, K. Shao, H. Sun, and B. Deng, “Trajectory tracking control of parallel manipulator based on udwadiakalaba approach," Mathematical Problems in Engineering, vol. 2017, Article ID 8975743, 12 pages, 2017. 


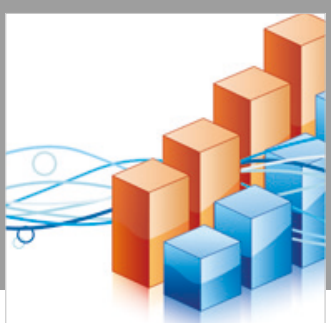

Advances in

Operations Research

\section{-n-m}
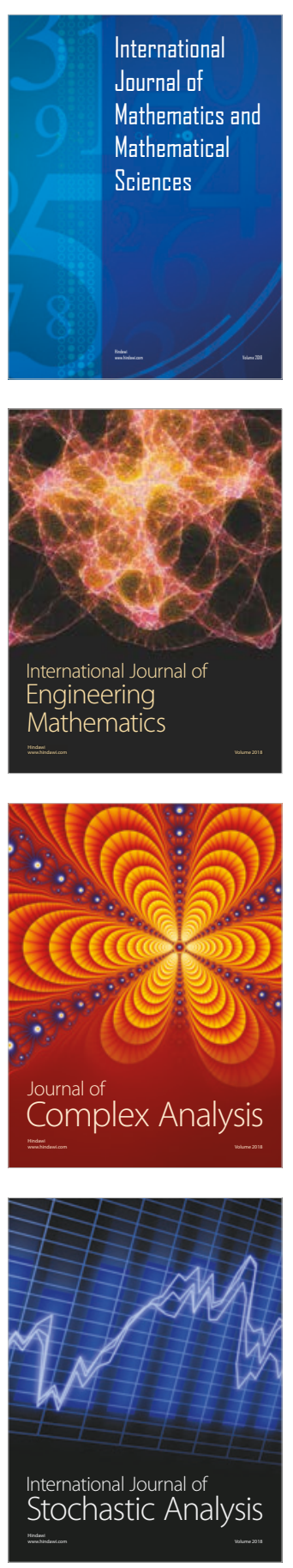
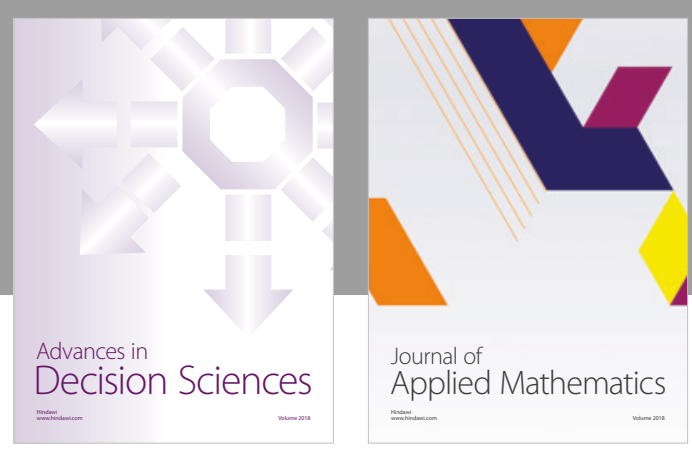

Journal of

Applied Mathematics
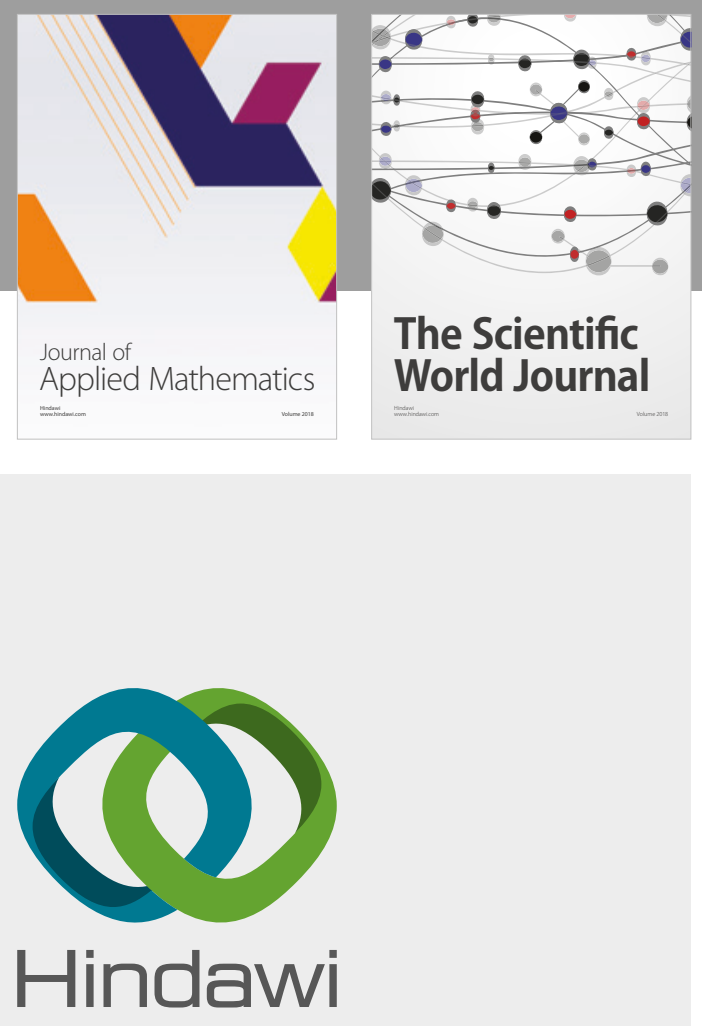

Submit your manuscripts at

www.hindawi.com

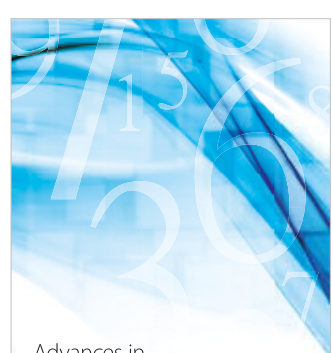

Advances in
Numerical Analysis
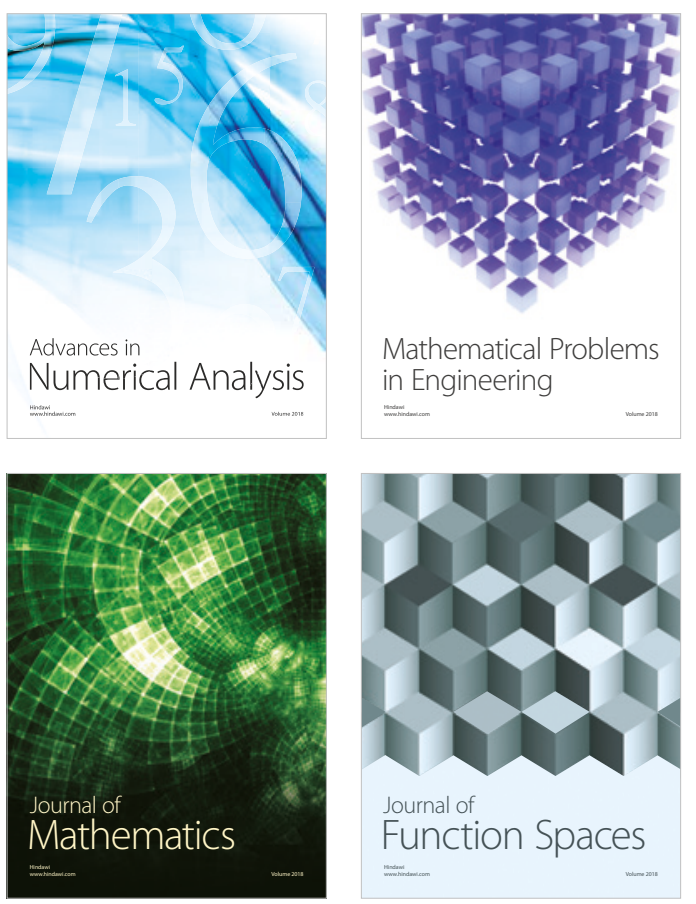

Mathematical Problems in Engineering

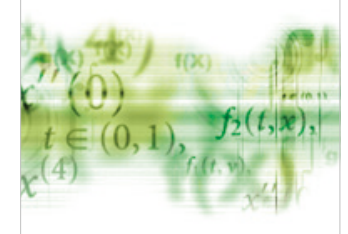

International Journal of

Differential Equations

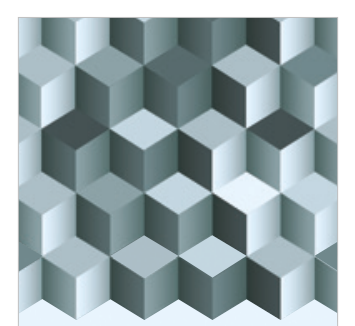

Journal of

Function Spaces

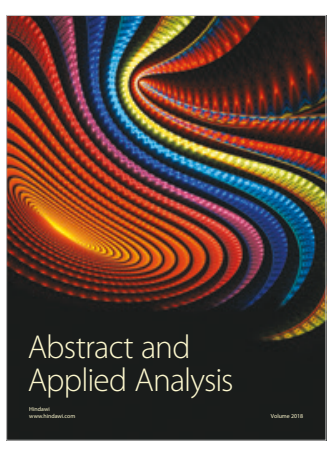

The Scientific

World Journal

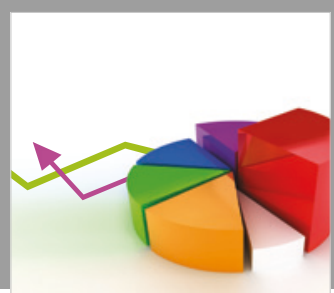

Journal of

Probability and Statistics
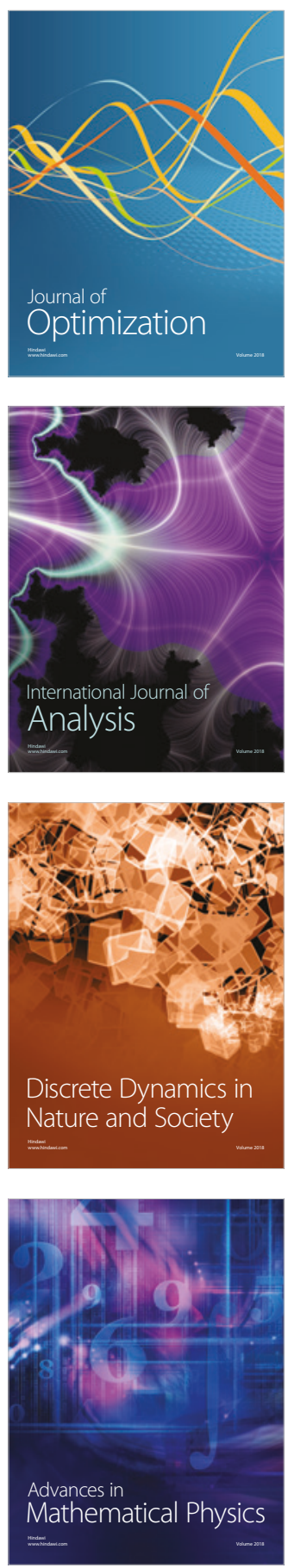